\title{
A importância do profissional enfermeiro na prevenção do HPV na Atenção Básica
}

\author{
The importance of professional nurses in the prevention of HPV in Primary Care \\ La importancia del enfermero profesional en la prevención del VPH en Atención Primaria
}

Recebido: 10/08/2021 | Revisado: 19/08/2021 | Aceito: 23/08/2021 | Publicado: 24/08/2021

\author{
Amanda Nicoly Hahn de Oliveira \\ ORCID: https://orcid.org/0000-0002-7200-8162 \\ Instituto Brasileiro de Medicina e Reabilitação, Brasil \\ E-mail: amandahahn17@gmail.com \\ Carla Carolini Capelini Zuleta \\ ORCID: https://orcid.org/0000-0002-9082-2774 \\ Instituto Brasileiro de Medicina e Reabilitação, Brasil \\ E-mail: carlacapelini@hotmail.com \\ Fabíola Teixeira Rosa \\ ORCID: https://orcid.org/0000-0001-9742-2397 \\ Instituto Brasileiro de Medicina e Reabilitação, Brasil \\ E-mail: fabiolateixeirarosa@gmail.com \\ Helga Rocha Pitta Portella Figueiredo \\ ORCID: https://orcid.org/0000-0003-2899-1988 \\ Instituto Brasileiro de Medicina e Reabilitação, Brasil \\ E-mail: helgapitta@gmail.com \\ Glória Maria Costa Rodriguez \\ ORCID: https://orcid.org/0000-0002-4456-4575 \\ Universidade Anhembi Morumbi, Brasil \\ E-mail: glorinha_rodriguez@hotmail.com
}

\begin{abstract}
Resumo
Objetivo: Reunir o conhecimento da literatura sobre a importância do profissional enfermeiro na prevenção do HPV na atenção básica. Metodologia: Foi realizada uma revisão da literatura, do tipo descritiva, com caráter qualitativo, mediante materiais indexados nas plataformas LILACS, BDENF, SciELO, disponíveis pela BVS - Biblioteca Virtual em Saúde e Revista Enfermagem UERJ, após busca e aplicação dos critérios de inclusão e exclusão, foram selecionados um total de 15 artigos, publicados entre os anos de 2011 e 2021 e redigidos no idioma português. Resultados e Discussão: O enfermeiro possui papel protagonista na prevenção do HPV. Podem ser citados como fatores de risco para a contaminação por HPV o déficit em educação em saúde e a falha na gestão das UBSs para um atendimento holístico da população feminina. Sendo assim, a enfermagem tem importante papel na prevenção do HPV e pode utilizar estratégias de educação em saúde, de busca ativa e incentivo à vacinação. Conclusão: Ficou evidente que o cuidado das mulheres com o HPV na atenção básica é a consequência de um atendimento com acolhimento ineficaz e uma baixa propagação de informações, expressando falhas na gestão, problemas para estabelecer um fluxo assistencial adequado, necessidade de novas técnicas preventivas e educativas, e educação continuada para os profissionais de saúde.
\end{abstract}

Palavras-chave: HPV; Enfermeiro; Atenção básica; Saúde da mulher; Educação em saúde.

\begin{abstract}
Objective: To gather knowledge from the literature on the importance of professional nurses in the prevention of HPV in primary care. Methodology: A descriptive, qualitative literature review was carried out using materials indexed on the LILACS, BDENF, SciELO platforms, available from the VHL - Virtual Health Library and Revista Enfermagem UERJ, after searching and applying the inclusion and exclusion criteria, a total of 16 articles were selected, published between 2011 and 2021 and written in Portuguese. Results and Discussion: The nurse has a leading role in the prevention of HPV. The deficit in health education and the failure in the management of UBSs for a holistic care of women can be cited as risk factors for HPV contamination. Thus, nursing has an important role in HPV prevention and can use health education strategies, active search and encouragement of vaccination. Conclusion: It was evident that the care of women with HPV in primary care is the consequence of an ineffective care service and a low dissemination of information, expressing failures in management, problems to establish an adequate flow of care, the need for new preventive and educational techniques, and continuing education for health professionals.
\end{abstract}

Keywords: HPV; Nurse; Primary care; Women's health; Health education.

\section{Resumen}

Objetivo: recopilar conocimientos de la literatura sobre la importancia del enfermero profesional en la prevención del VPH en atención primaria. Metodología: Se realizó una revisión descriptiva, cualitativa de la literatura utilizando 
materiales indexados en las plataformas LILACS, BDENF, SciELO, disponibles en la BVS - Biblioteca Virtual en Salud y Revista Enfermagem UERJ, después de buscar y aplicar los criterios de inclusión y exclusión, un total de 16 artículos fueron seleccionados, publicados entre 2011 y 2021 y escritos en portugués. Resultados y discusión: La enfermera tiene un papel de liderazgo en la prevención del VPH. El déficit en educación para la salud y el fracaso en el manejo de SBU para una atención integral de la mujer pueden citarse como factores de riesgo de contaminación por VPH. Por tanto, la enfermería tiene un papel importante en la prevención del VPH y puede utilizar estrategias de educación sanitaria, búsqueda activa y fomento de la vacunación. Conclusión: Se evidenció que la atención de mujeres con VPH en atención primaria es consecuencia de un servicio de atención ineficaz y una baja difusión de información, expresando fallas en la gestión, problemas para establecer un adecuado flujo de atención, la necesidad de nuevas técnicas preventivas y educativas, y educación continua para profesionales de la salud.

Palabras clave: VPH; Enfermera; Atención primaria; Salud de la mujer; Educación para la salud.

\section{Introdução}

O HPV (sigla em inglês para papilomavírus humano) é um vírus que infecta epitélios escamosos e pode induzir uma grande variedade de lesões cutaneomucosas, estas lesões podem preceder o câncer de colo de útero (Ministério da Saúde, 2015). A fim de promover a saúde e a prevenção de doenças ou enfermidades, como o HPV, o enfermeiro na atenção básica visa incluir intervenções como a educação em saúde sobre estilos de vida saudáveis com o objetivo de diminuir os agravos decorrentes desta infecção (Brunner, 2014).

Define-se atenção básica como um "conjunto de ações de saúde individuais, familiares e coletivas que envolvem promoção, prevenção, proteção, diagnóstico, tratamento, reabilitação, redução de danos, cuidados paliativos e vigilância em saúde, desenvolvida por meio de práticas de cuidado integrado e gestão qualificada, realizada com equipe multiprofissional e dirigida à população em território definido, sobre as quais as equipes assumem responsabilidade sanitária." (Ministério da Saúde, 2012). Wanda Horta, em 1970 definiu a enfermagem como uma ciência é uma arte, cabendo a ela desenvolver atividades para a manutenção e promoção da saúde, bem como para a prevenção de doenças sendo de sua responsabilidade o diagnóstico e a intervenção de enfermagem (Horta, 1979). Neste contexto, o enfermeiro atua diretamente no diagnóstico, prevenção e tratamento do HPV por meio das consultas de enfermagem onde é realizado processo de Sistematização da Assistência da Enfermagem. Portanto, delimita-se como questão norteadora deste trabalho: Qual a importância do profissional enfermeiro frente a prevenção do HPV na atenção básica?

A transmissão do HPV acontece prioritariamente por qualquer tipo de atividade sexual e, excepcionalmente, durante o parto (Ministério da Saúde, 2015). O Ministério da Saúde preconiza que toda mulher entre 25 e 64 anos de idade, que já iniciou sua vida sexual, deve se submeter ao exame preventivo, com periodicidade anual, inicialmente (INCA, 2016). Existem mais de 200 tipos diferentes de HPV, dentre esses, quatro tipos são mais frequentes. Os HPV tipos 16 e 18 causam a maioria dos casos de câncer de colo do útero em todo o mundo. Já os tipos 6 e 11 causam a maioria das verrugas genitais (Ministério da Saúde, 2015). A infecção por um determinado tipo de HPV não impede a infecção por outros tipos, podendo ocorrer infecção múltipla. Na maioria das pessoas, o HPV não produz qualquer manifestação e o tempo de latência pode variar de meses a anos e, quando presentes, as manifestações podem ser subclínicas (Ministério da Saúde, 2015). A prevenção do papilomavírus humano, na atenção integral à saúde da mulher, também é uma prática dos profissionais de enfermagem, e cabe a esses profissionais realizar a consulta de enfermagem, o exame preventivo, solicitar exames complementares e prescrever medicações, conforme protocolos ou outras normativas técnicas estabelecidas pelo gestor municipal, observadas as disposições legais da profissão (Ministério da Saúde, 2013).

Recentemente, o Ministério da Saúde (MS) por meio do Programa Nacional de Imunizações (PNI) instituiu uma nova estratégia de prevenção. Duas vacinas contra o HPV foram aprovadas para uso, a bivalente e a quadrivalente, se forem administradas antes da exposição ao vírus elas previnem mais de 95\% das infecções. De acordo com a ANVISA, a vacina quadrivalente é aprovada para a faixa etária de mulheres entre 9-45 anos e homens entre 9-26 anos e a vacina bivalente para 
mulheres entre 10-25 anos. O Ministério da Saúde e o SUS oferecem a vacina quadrivalente, que previne contra o HPV dos tipos 6,11,16,18, para meninas entre 9-14 anos e meninos entre 11-14 anos em 2 duas doses com 6 meses de intervalo entre elas. Em 2021, o Ministério da Saúde ampliou a vacinação oferecida pelo SUS para mulheres imunossuprimidas de até 45 anos vivendo com HIV/Aids, transplantadas e portadoras de cânceres. Seguindo a orientação da OMS, o esquema será feito com a aplicação de 3 doses em intervalos de 2 meses entre a primeira e a segunda, e a terceira dose 6 meses após a primeira aplicação. (Ministério da Saúde, 2021).

Em consonância com o contexto exposto, esse trabalho tem o objetivo geral de reunir o conhecimento da literatura sobre a importância do profissional enfermeiro na prevenção do HPV na atenção básica. Segundo um estudo realizado sobre a prevalência de infectados pelo HPV em 2017, 83,4\% dos entrevistados apresentaram comportamento sexual de risco (Associação Hospitalar Moinhos de Vento, 2017). O aumento dos números de casos de câncer de colo de útero esperados para o Brasil em 2019, será de 16.590 casos para cada ano do triênio 2020-2022, com um risco estimado de 15,43 a cada 100 mil mulheres (INCA, 2019). Acredita-se que esta pesquisa irá contribuir para assegurar uma melhor prática da Sistematização da Assistência da Enfermagem pelos enfermeiros na prevenção do HPV, além de procurar estimular a educação em saúde.

\section{Metodologia}

Trata-se de uma revisão integrativa de literatura que surgiu das seguintes etapas de elaboração: definição do tema e questão norteadora, estabelecimento de critérios de inclusão e exclusão, definição das informações a serem extraídas dos estudos, avaliação dos estudos, interpretação dos principais resultados e a elaboração do documento que contempla todas essas fases.

A revisão integrativa proporciona aos profissionais de saúde um rápido acesso aos resultados de pesquisas pertinentes às condutas e às tomadas de decisão, proporcionando um saber crítico, contribuindo para melhorias no cuidado prestado ao paciente e familiar, fomentando a passagem de novos saberes para a prática. (MENDES, 2008)

O levantamento dos dados foi realizado no mês de março de 2021. As bases de dados pesquisadas foram: Literatura Latino- Americana e do Caribe em Ciências da Saúde (LILACS), Base de Dados de Enfermagem (BDENF), Biblioteca Eletrônica Scientific Electronic Library Online (SciELO), disponíveis na BVS - Biblioteca Virtual em Saúde e na Revista Enfermagem UERJ, utilizando os seguintes descritores: HPV, Enfermeiro, Atenção Básica, Saúde da Mulher, Enfermagem, Educação em Saúde, Enfermagem em Saúde Comunitária, Prevenção Primária e Promoção da saúde. Esses descritores foram inicialmente consultados em Descritores em Ciência da Saúde (DeCS). Os critérios de inclusão utilizados foram: artigos científicos completos, idioma em português, publicados entre os anos de 2011 e 2021. Foram excluídos os artigos incoerentes com a questão norteadora e que não estavam disponíveis eletronicamente. Após o levantamento das publicações, os títulos e resumos foram lidos e analisados segundo os critérios de exclusão e inclusão estabelecidos e que trouxeram contribuições importantes para o desenvolvimento do estudo. Depois de analisados, obteve-se a amostra final de 15 artigos de um total de 45 achados. 
Fluxograma 1. Busca e seleção dos artigos. Rio de Janeiro, RJ, Brasil, 2021.
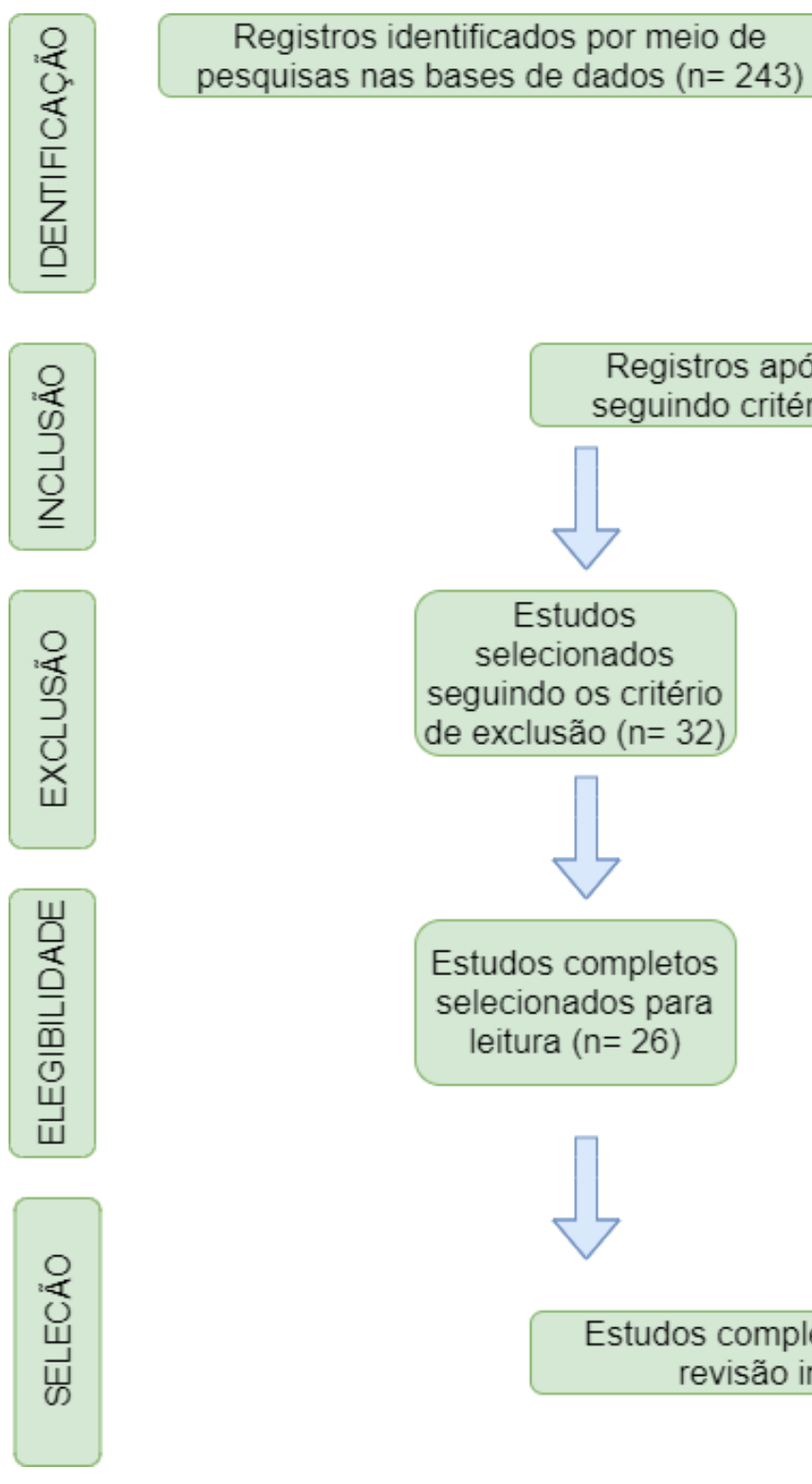

Registros identificados por meio de pesquisas em outras fontes de dados $(n=0)$

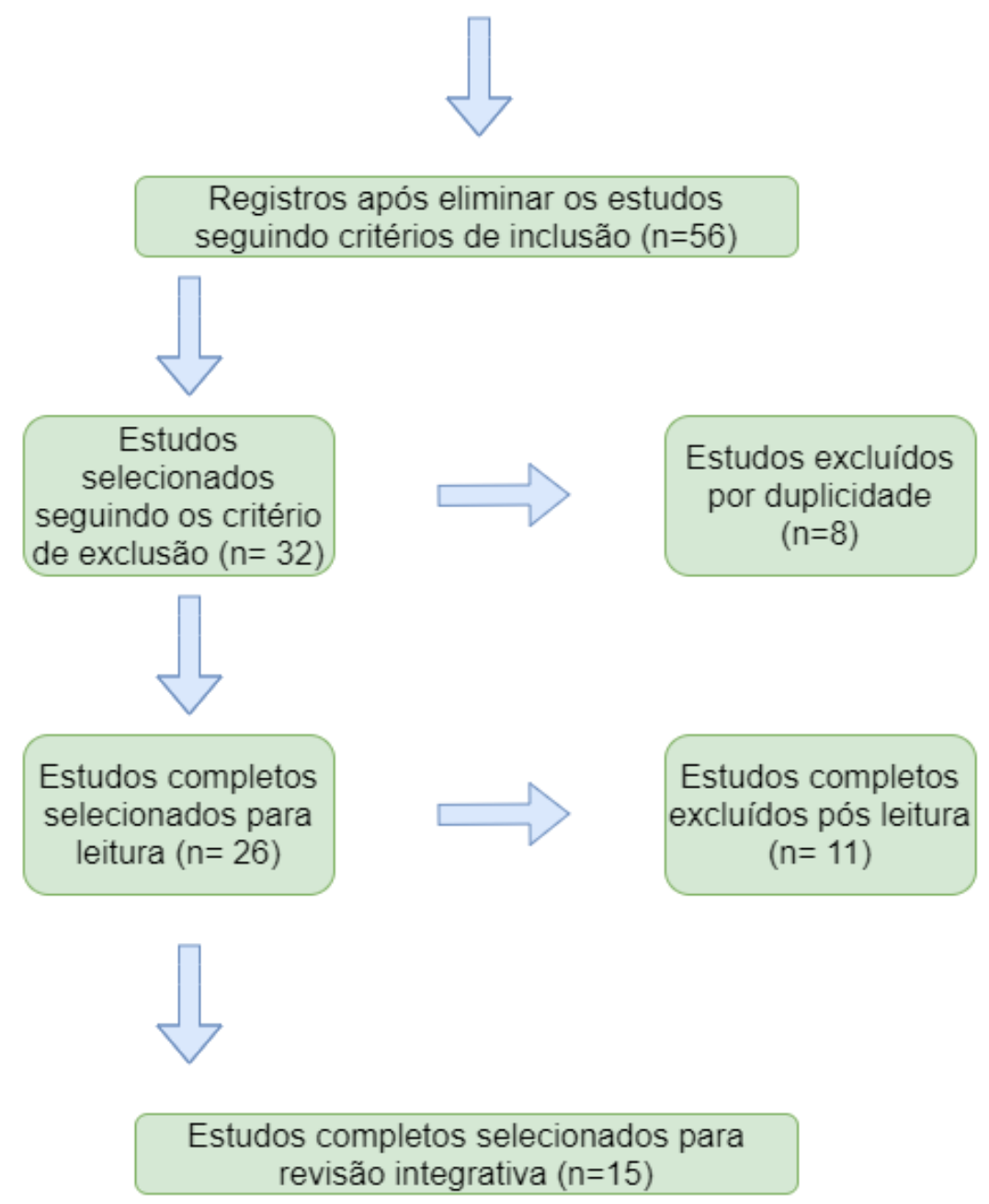

Fonte: Dados da pesquisa.

\section{Resultados}

No quadro abaixo apresentaremos a síntese de todos os estudos incluídos na pesquisa e que representaram a base para elaboração dos resultados, discussão e conclusão sobre a importância do enfermeiro na prevenção do HPV na atenção básica. Dos quinze artigos selecionados no campo deste estudo, todos tiveram publicação entre 2012 e 2020, sendo em maior quantidade as apresentações dos artigos de 2014 e 2017. A partir dos resultados foi possível dividir a sistematização da assistência de enfermagem entre os temas abordados dentro dos artigos nas temáticas principais, foram elas: atuação da enfermagem frente aos fatores de risco relacionados ao HPV na atenção básica de saúde, atuação da enfermagem frente a prevenção relacionada ao HPV na atenção básica de saúde, atuação da enfermagem frente ao machismo, tabus e sexualidade relacionados ao HPV, atuação da enfermagem frente às condições emocionais relacionadas ao HPV. 
Quadro 1. Quadro expositivo dos artigos pesquisados neste estudo. Rio de Janeiro, RJ, Brasil, 2021

\begin{tabular}{|c|c|c|}
\hline AUTOR/ANO & TÍTULO & OBJETIVOS \\
\hline $\begin{array}{l}\text { Dalmacio, N.C.G.; Souza da Costa, B. } \\
\text { E.;Souza, S.C.S.; Aguiar, V.F.F. - } \\
2019\end{array}$ & $\begin{array}{l}\text { Percepção da mulher com HPV e } \\
\text { seu autocuidado. }\end{array}$ & $\begin{array}{l}\text { Descrever a percepção das mulheres acometidas por HPV } \\
\text { em relação à sua situação de saúde e aos tipos de práticas } \\
\text { para o autocuidado desenvolvidos pela mulher, baseando- } \\
\text { se na Teoria do Autocuidado de Orem. }\end{array}$ \\
\hline $\begin{array}{l}\text { Carvalho, M.C.M.P.; Queiroz, } \\
\text { A.B.A.; Moura, M.A.V.; Marques, } \\
\text { S.C.; Vieira, B.D.G.; Ferreira, D.C.. - } \\
2017\end{array}$ & $\begin{array}{l}\text { Fatores de risco de mulheres } \\
\text { adolescentes e jovens frente ao } \\
\text { papilomavírus humano. }\end{array}$ & $\begin{array}{l}\text { Identificar os fatores de risco à infecção pelo } \\
\text { Papilomavírus Humano (HPV) associados aos } \\
\text { comportamentos e atitudes de adolescentes e jovens de uma } \\
\text { unidade escolar de Ensino Médio do Rio de Janeiro. }\end{array}$ \\
\hline Souza, A.F.; Costa, L.H.R.. - 2015 & $\begin{array}{l}\text { Conhecimento de mulheres sobre } \\
\text { HPV e câncer de colo do útero } \\
\text { após consulta de enfermagem. }\end{array}$ & $\begin{array}{l}\text { Compreender a capacidade de assimilação das mulheres } \\
\text { que realizam o exame Papanicolaou acerca do } \\
\text { papilomavírus humano e sua relação com o câncer do colo } \\
\text { do útero, por meio das informações e/ou orientações } \\
\text { repassadas durante a consulta realizada por enfermeiros. }\end{array}$ \\
\hline $\begin{array}{l}\text { Cestari, M.E.W.; Merighi, M.A.B.; } \\
\text { Garanhani, M.L.; Cardeli, A.A.M.; } \\
\text { Jesus, M.C.P.; Lopes, D.F.M - } 2012\end{array}$ & $\begin{array}{l}\text { Necessidades de cuidados de } \\
\text { mulheres infectadas pelo } \\
\text { papilomavírus humano: uma } \\
\text { abordagem compreensiva. }\end{array}$ & $\begin{array}{l}\text { Objetivou compreender as necessidades de cuidados das } \\
\text { mulheres infectadas pelo Papilomavírus Humanos. }\end{array}$ \\
\hline $\begin{array}{l}\text { Silva, M.M; Gitsos, J.; Santos, N.L.P. } \\
\text { - } 2013\end{array}$ & $\begin{array}{l}\text { Atenção básica em saúde: } \\
\text { prevenção do câncer de colo do } \\
\text { útero na consulta de enfermagem. }\end{array}$ & $\begin{array}{l}\text { Objetivou analisar os eixos teórico-conceituais } \\
\text { estruturantes da consulta de enfermagem ginecológica na } \\
\text { AB; e discutir as principais condutas implementadas para } \\
\text { prevenção do CCU. }\end{array}$ \\
\hline $\begin{array}{l}\text { Santiago, T.R.; Andrade, M.S.; } \\
\text { Paixão, G.P.N. - } 2014\end{array}$ & $\begin{array}{l}\text { Conhecimento e prática das } \\
\text { mulheres atendidas na unidade de } \\
\text { saúde da família sobre o } \\
\text { papanicolau }\end{array}$ & $\begin{array}{l}\text { Objetivo de descrever o conhecimento e a prática sobre o } \\
\text { Papanicolaou das mulheres, entre } 25 \text { e } 59 \text { anos, residentes } \\
\text { na área de abrangência da Unidade de Saúde da Família } \\
\text { (USF) Nossa Senhora de Fátima do Alto da Maravilha, em } \\
\text { Senhor do Bonfim, Bahia (BA). }\end{array}$ \\
\hline $\begin{array}{l}\text { Santos, L.V.; Inagaki,A.D.M.; Abud, } \\
\text { A.C.F.; Oliveira, J.K.A.; Ribeiro, } \\
\text { C.J.N.; Oliveira, M.I.A. - } 2014\end{array}$ & $\begin{array}{l}\text { Características sociodemográficas } \\
\text { e risco para doenças sexualmente } \\
\text { transmissíveis entre mulheres } \\
\text { atendidas na atenção básica. }\end{array}$ & $\begin{array}{l}\text { Objetivou traçar o perfil sociodemográfico de mulheres que } \\
\text { realizaram exame ginecológico no período de janeiro de } \\
2009 \text { a junho de 2012, bem como levantar os fatores de } \\
\text { risco desta população para as doenças sexualmente } \\
\text { transmissíveis (DST). }\end{array}$ \\
\hline $\begin{array}{l}\text { Dantas, P.V.J.; Leite, K.N.S.; César, } \\
\text { E.R.S.; Silva, S.C.R.; Souza, T.A.; } \\
\text { Nascimento, B.B. - } 2018\end{array}$ & $\begin{array}{l}\text { Conhecimento das mulheres e } \\
\text { fatores da não adesão acerca do } \\
\text { exame papanicolau }\end{array}$ & $\begin{array}{l}\text { Objetivos: Averiguar o conhecimento das mulheres sobre o } \\
\text { Papanicolau; Mostrar os fatores que colaboram para a não } \\
\text { adesão ao Papanicolau; Identificar as orientações de } \\
\text { enfermagem sobre o Papanicolau. }\end{array}$ \\
\hline Santos, U.M.; Souza, S.E.B. - 2013 & $\begin{array}{l}\text { Papanicolau: diagnóstico precoce } \\
\text { ou prevenção do câncer cervical } \\
\text { uterino? }\end{array}$ & $\begin{array}{l}\text { Objetivo primário de discutir a importância do exame } \\
\text { Papanicolaou na prevenção do câncer de colo do útero e, } \\
\text { como objetivo secundário, identificar os fatores que } \\
\text { dificultam a realização do Papanicolaou. }\end{array}$ \\
\hline $\begin{array}{l}\text { Silva, L.S.R.; Lessa, E.C.; Silva, } \\
\text { T.M.; Albuquerque, A.K.D.S.; } \\
\text { Ferreira, M.D.R.; Silva, T.L.L. - } 2016\end{array}$ & $\begin{array}{l}\text { Adesão ao exame papanicolau por } \\
\text { mulheres jovens em unidade } \\
\text { básica de saúde. }\end{array}$ & $\begin{array}{l}\text { Investigar os motivos do não comparecimento ao exame } \\
\text { preventivo Papanicolau das mulheres jovens que realizaram } \\
\text { o exame em 2012, mas não houve a repetição em } 2013 \text {. }\end{array}$ \\
\hline $\begin{array}{l}\text { Conceição, J.P.S.; Medeiros, M.M.S.; } \\
\text { Rodrigues, L.M.S.; Bráz, M.R.; } \\
\text { Balbino, C.M.; Silvino, Z.R. - } 2017\end{array}$ & $\begin{array}{l}\text { O conhecimento do enfermeiro } \\
\text { sobre a prevenção do câncer de } \\
\text { colo de útero na atenção básica. }\end{array}$ & $\begin{array}{l}\text { Objetiva-se identificar o conhecimento do enfermeiro da } \\
\text { Atenção Básica quanto à prevenção do câncer de colo de } \\
\text { útero, descrever a percepção do enfermeiro quanto à } \\
\text { prevenção deste câncer, e identificar as atividades de } \\
\text { prevenção realizadas pela Atenção Básica de Saúde. }\end{array}$ \\
\hline
\end{tabular}




\begin{tabular}{|l|l|l|}
\hline $\begin{array}{l}\text { Guedes, M.C.R.; Bento, P.A.S.S.; } \\
\text { Telles, A.C.; Queiroz, A.B.A.; } \\
\text { Xavier, R.B. - 2017 }\end{array}$ & $\begin{array}{l}\text { A vacina do papilomavírus } \\
\text { humano e o câncer do colo do } \\
\text { útero: uma reflexão }\end{array}$ & $\begin{array}{l}\text { Promover reflexões sobre a vacina contra o papilomavírus } \\
\text { humano relacionadas à baixa adesão. }\end{array}$ \\
\hline $\begin{array}{l}\text { Feitosa, L.G.; Alves, D.L.; Pereira, } \\
\text { E.C.T.; Nunes, V.R.; Hipólito, U.V.; } \\
\text { Almeida, M.C.S. - 2019 }\end{array}$ & $\begin{array}{l}\text { Imunização contra papilomavírus } \\
\text { humano em escolas municipais }\end{array}$ & $\begin{array}{l}\text { Descrever a experiência da realização de atividades } \\
\text { educativas, análise do estado vacinal e imunização contra o } \\
\text { papilomavírus humano (HPV) em estudantes da rede } \\
\text { municipal da região norte de Palmas - TO. }\end{array}$ \\
\hline $\begin{array}{l}\text { Silva, P.L.N.; Martins, F.G.S.; } \\
\text { Galvão, A.P.F.C.; Souto, S.G.T.; }\end{array}$ & $\begin{array}{l}\text { Sentimentos de pré-adolescentes e } \\
\text { adolescentes quanto à vacinação } \\
\text { contra o papilomavírus humano }\end{array}$ & $\begin{array}{l}\text { Identificar os sentimentos de pré-adolescentes e } \\
\text { adolescentes quanto à vacinação contra o papilomavírus } \\
\text { humano. }\end{array}$ \\
\hline $\begin{array}{l}\text { Oliveira,V.C.; Silva, M.R.; Viegas, } \\
\text { S.M.F.; Guimarães, E.A.A.; Fonseca, } \\
\text { D.F.; Oliveira, P.P. - 2020 }\end{array}$ & $\begin{array}{l}\text { Vivência de responsáveis por } \\
\text { adolescentes na vacinação contra } \\
\text { o papilomavírus: estudo } \\
\text { fenomenológico }\end{array}$ & $\begin{array}{l}\text { Compreender a vivência de responsáveis por adolescentes } \\
\text { em relação a vacinação contra o papilomavírus humano. }\end{array}$ \\
\hline
\end{tabular}

Fonte: Dados da pesquisa.

\section{Discussão}

\subsection{Atuação da Enfermagem frente aos fatores de risco relacionados ao HPV na atenção básica de saúde}

De acordo com Carvalho, et al. 2017, o início precoce da vida sexual e a multiplicidade de parceiros são fatores de risco para a infecção pelo papilomavírus humano (HPV), assim como a estabilidade conjugal, pois esta implica na diminuição ou até mesmo eliminação do uso de preservativos. Souza et al. 2015, complementa dizendo que é muito importante que a educação primária em saúde seja direcionada especialmente para população com parceiro fixo pois são os mais vulneráveis ao contágio pelo vírus.

Santos et al. 2014 afirma que o início precoce da vida sexual está intrinsecamente ligado ao não uso de preservativos, e associados à uma baixa condição social são considerados como principais fatores de risco pelo autor.

Outros dois autores também afirmam que a estabilidade conjugal é um fator de risco (Cestari et al. 2012), relatando que tanto as mulheres com múltiplos parceiros, quanto as mulheres com um parceiro fixo, não realizam a prática adequada do exame preventivo. (Santiago, et al. 2014).

Ressaltamos que o HPV pode ter um período de incubação de meses ou até anos, sendo assim, não é possível determinar se o contágio é recente ou antigo. A estabilidade conjugal, apesar de parecer um fator de segurança em decorrência de uma confiança mútua entre parceiros, acreditamos que se torne um fator de risco somando-se a dificuldade de aceitação do uso de preservativos.

De acordo com Silva, et al. 2016, um bom nível de escolaridade torna as mulheres mais flexíveis a receber novos conhecimentos e consequentemente aderir melhor ao exame de Papanicolau. Já Santiago afirma que mesmo com baixa escolaridade a prática do exame Papanicolau foi adequada. Em relação ao HPV propriamente dito, mesmo mulheres com baixa escolaridade, em sua maioria associam o HPV com o CCU (Câncer de Colo de Útero) (Dalmacio, et al. 2019).

Geralmente quando a mulher descobre que está contaminada com Papilomavírus Humano, logo o associa ao câncer de colo de útero ficando aflita. Em contrapartida, outras mulheres por falta de informação, não associam o HPV a algo grave, deixando de fazer o preventivo e o acompanhamento rotineiro. De acordo com o estudo de Santiago, et al. 2014, grande parcela das mulheres está mais preocupada com uma possível gestação do que uma possível contaminação pelo HPV, o que explica o motivo da pílula anticoncepcional ser o contraceptivo mais utilizado. Sabe-se que o uso apenas do anticoncepcional sem estar associado ao preservativo é um fator que gera riscos à saúde pois não previne contra ISTs dando assim uma falsa segurança para 
a mulher, além deste método, não ser $100 \%$ seguro para prevenção da gravidez. Acreditamos então, que quanto menos conhecimento sobre saúde, direitos sexuais e reprodutivos as mulheres possuírem, menores serão as chances de haver uma mudança significativa na saúde pública, na saúde sexual e nos índices de infecções pelo HPV.

O fator socioeconômico está diretamente ligado à disponibilidade para acompanhamento e tratamento das ISTs, assim como para a compreensão de sua gravidade e modos de prevenção. Criando assim brechas para que futuramente possa haver uma evolução do HPV para o CCU por não acontecer uma periodicidade de cuidados preventivos. Expressa-se assim, a carência de uma boa base de informações, mostrando a necessidade do enfermeiro na atenção básica de saúde para suprir essa carência.

Em seu estudo, Santiago et al. 2014 relata que as mulheres que mais realizam o exame preventivo de maneira adequada são as com idade mais avançada, entre 40 a 59 anos de idade, evidenciando que com o passar da idade também se somam as experiências de vida e uma maior educação em saúde.

É importante que as mulheres que não realizam o exame preventivo, sejam prioridade na busca ativa, através da visita domiciliar estabelecendo vínculo com a unidade de saúde. A busca ativa realizada em escolas e igrejas também é uma alternativa eficiente para que esta seja realizada de forma mais abrangente, pois atinge mais mulheres do que nas UBS. Sendo neste momento, muito importante a figura do Agente Comunitário de Saúde (ACS) em parceria com a enfermagem (Conceição et al. 2017).

Oliveira, et al. 2020, revalida que os fatores que mais afetam a credibilidade do sistema básico de saúde são a falta de materiais, falta de sensibilidade e tratamento humanizado dos profissionais, e falta de pessoal qualificado para lidar com a população. A falta de continuidade do tratamento não está ligada apenas ao abandono das mulheres, está relacionada também com a falta de profissionais nas UBS, ou até mesmo abandono do posto de saúde durante o expediente. O que evidencia os obstáculos para uma gestão de qualidade. (Santos, et al. 2013).

Percebe-se que a falha da gestão causa problemas para estabelecer um fluxo assistencial adequado, já que cada unidade de saúde tem suas peculiaridades. Atribui-se a isso a falta de continuidade no tratamento de doenças nas mulheres, sendo uma problemática dos princípios e diretrizes que regem o Sistema Único de Saúde (SUS).

A partir dessas discussões, percebe-se a necessidade de novas técnicas preventivas e educativas na participação do enfermeiro como profissional de saúde da atenção básica, visando a eficácia no incentivo populacional das práticas adequadas de saúde, reconhecendo e levando em consideração os aspectos emocionais, socioeconômicos e biológicos, que levam ao acometimento do HPV. (Carvalho, et al. 2017). As orientações passadas para essas mulheres precisam abranger todos os aspectos das ISTs e não priorizar somente o exame citopatológico. Essa é uma forma de educar essa mulher para que a repetição da infecção não aconteça. Dessa maneira, pode-se ressaltar a grande importância que a enfermagem tem diante da educação em saúde, pois é o profissional que mais tem contato no cuidado primário, devendo estimular a autonomia da paciente através de conhecimentos passados, nunca deixando de lado o vínculo, humanização e empatia, pois são ferramentas da tecnologia leve, muito importantes para a realização de um ótimo atendimento prestado. Levando sempre nos atendimentos a teoria de Myra Levine (1967), que enfatiza a importância de um atendimento holístico, afirmando que a essência da enfermagem é a interação humana.

\subsection{Atuação da Enfermagem frente a prevenção relacionada ao HPV na atenção básica de saúde}

No estudo de Santiago et al. 2014 mais de 80\% das entrevistadas não detinham o conhecimento certo sobre a real finalidade do exame Papanicolau. Adicionalmente, Santos, et al. 2014 relata que as mulheres só procuram a UBS quando manifestam algum sintoma ginecológico. Silva, et al. 2016 complementa dizendo que um dos motivos das mulheres não realizarem o exame seria estarem bem de saúde evidenciando o modelo curativista ainda predominante desta população. É importante enfatizar que o exame papanicolau serve para a detecção do HPV e não para a sua prevenção, sendo essa realizada através da vacinação, preservativos e abstinência sexual (Santos et al. 2013). As mulheres necessitam de um bom entendimento 
sobre a infecção, para que as medidas preventivas sejam acatadas. Essa afirmativa é reforçada quando Feitosa et al. 2019, relata que a prevenção é a melhor forma de controle contra a infecção por HPV. É importante ressaltar que o papanicolau não envolve somente a coleta citopatológica, mas também envolve indiretamente o exame físico na consulta ginecológica de enfermagem, sendo este muito importante para detectar condilomas aculminados provenientes do HPV.

Santos et al. 2013 explicita outro fator importante que aparece na realidade das consultas de enfermagem nas UBS, que é o material coletado para o exame de forma imprópria, o que prejudica a precisão do resultado. Esse fato reflete o despreparo dos profissionais que podem levar a um falso diagnóstico e consequentemente desconfiança por parte das clientes.

Faz-se necessário haver uma efetiva educação continuada para os profissionais de saúde, em especial ao enfermeiro que realiza a coleta do exame Papanicolau nas UBS, para que não aconteçam erros de coleta e para que as orientações em relação à prevenção sejam feitas de forma precisa e eficaz. Essas recomendações podem ser implementadas individualmente nas consultas de enfermagem enfatizando a importância de realizar o exame independente de sua orientação sexual e independente de sua situação conjugal, além disso faz-se necessário destacar a importância do retorno dessa mulher para receber os resultados dos exames.

Não menos importante, o uso de preservativo ainda é uma das melhores formas de prevenção, Carvalho et al. 2017 diz que os jovens não são adeptos ao mesmo em seu cotidiano, deixando-os assim mais vulneráveis à infecção pelo papilomavírus humano. Dalmacio et al. 2019 afirma em sua pesquisa que a maioria das garotas nunca usou preservativo, e as poucas que usaram, só mencionaram a camisinha masculina em sua primeira relação sexual. Já os garotos têm uma porcentagem menor ainda, principalmente na primeira relação sexual. A maior parte das adolescentes acredita que só de usar o preservativo já está automaticamente livre de contágio pelo HPV. Vale destacar que é errada a ideia de que apenas com o uso do preservativo se evita a infecção pelo HPV, pois o preservativo apenas minimiza as chances de contaminação, ou seja, não é uma garantia de prevenção. Deixar fora de questão a camisinha feminina é um erro, pois é o método que mais protege do contágio pelo vírus do HPV nas relações sexuais, por cobrir a maior parte da mucosa vaginal. É muito importante esclarecer que o uso do preservativo não deve ser só na penetração, mas sim em todas as preliminares como no sexo oral também.

Silva et al. 2020, afirma que a prevenção contra ISTs se dá por meio da conscientização destes jovens sobre a importância dos cuidados necessários com a saúde sexual e propõe estratégias conjuntas entre pais, professores e profissionais da saúde para a transmissão dessas informações.

Para isso, faz-se necessário atentar-se sobre os principais meios de informações entre os jovens, em especial os meios digitais, principalmente as redes sociais, onde nos dias atuais é o meio mais utilizado de propagação de informações.

Pode-se perceber uma deficiência por parte da atenção básica em atender a demanda dessa população mais jovem, por isso a proposta acima pode ser complementada com o uso das redes sociais como forma de propagação das informações por parte dos profissionais de saúde, profissionais da educação e pelos próprios pais em casa, desmistificando a ideia de que toda a responsabilidade de educação sexual e educação em saúde deve ser realizada apenas por profissionais da saúde, atribuindo caráter de responsabilidade para toda rede de apoio desses jovens.

Dalmacio, et al. 2019 enfatiza a importância de se orientar a respeito da vacina, já que essa previne contra os tipos oncogênicos do HPV. Guedes, et al. 2017, problematiza o fato da vacina não ser rotineira e não estar no calendário vacinal, o que gera uma não aceitação da mesma por parte da população. A discussão precoce contribui para a conscientização sobre a vacina e por isso faz necessário propor estratégias para a transmissão dessas informações (Silva et al. 2020). Fica claro que a discussão sobre a vacina do HPV ainda é cercada pela falta de informação e compreensão equivocada sobre a sua real importância. O modo como se transmitem essas informações, se tratando de um assunto polêmico e novo como a vacinação contra o HPV, pode impactar no processo decisório de vacinar, então o enfermeiro, como profissional protagonista na sala de vacinação deve se atentar aos diversos fatores que contribuem para não aceitação da vacina tanto pelos pais quanto pelos adolescentes. Fatores 
como o medo, dificuldade de diálogo com um público jovem, estigma de que a vacina é uma carta branca para o início da vida sexual, devem ser combatidos pelos profissionais de saúde junto com o governo, através de propostas mais tecnológicas, como educação em saúde através das redes sociais.

Feitosa, et al. 2019 relata que a vacinação contra o HPV das meninas é realizada em maior quantidade em relação aos meninos. Porém, não relata os reais motivos disso acontecer, o que configura uma limitação ao fazer a análise deste tema.

Oliveira, et al. 2020 complementa destacando a importância da vacinação acontecer na mesma idade para ambos os sexos, destacando que os homens começam a vida sexual antes das mulheres, elencando o fato dos homens serem um dos principais transmissores do HPV.

Não tão menos importante quanto o incentivo para a vacinação, a orientação quanto ao uso de preservativos e a realização adequada da coleta citológica preventiva devem ser feitas constantemente e insistentemente, pois junto com a abstinência sexual ainda são as melhores formas de prevenção contra a infecção pelo Papilomavírus Humano e controle das lesões induzidas pelo HPV, evitando o desenvolvimento do CCU.

Como a busca das mulheres nos serviços de saúde para as ações de prevenção não são efetivas, o profissional enfermeiro tem um papel fundamental e é um dos responsáveis na conscientização para prevenção do HPV, utilizando as oportunidades de qualquer encontro com essas mulheres para transmitir as informações necessárias. (Conceição, et al. 2017)

\subsection{Atuação da Enfermagem frente ao machismo, tabus e sexualidade relacionados ao HPV}

Quando o assunto é sexualidade feminina, o tema também vem acompanhado de muitos obstáculos de gênero, o qual precisa ser discutido de forma responsável levando em conta que a sexualidade das mulheres ainda é um tabu em uma sociedade patriarcal (Guedes et al. 2017). Já Souza et al. 2015, enfatiza a dificuldade que as mulheres têm em relação ao uso do preservativo relacionando isso à submissão, vergonha e ao medo.

É normal que as mulheres muitas vezes cresçam sem a devida educação sexual e reprodutiva e consequentemente, não tenham pleno conhecimento e informações sobre seus direitos reprodutivos e sexuais quando chegam à UBS. Em uma sociedade onde o machismo estrutural é bastante evidenciado, padrões de comportamentos são sempre esperados de ambos os gêneros, o desafio da educação sobre sexualidade está justamente aí, pois não basta passar as informações e organizar estratégias paras que elas cheguem ao público-alvo, mas sim de como fazer esse público alvo absorver as informações ao ponto de elas se tornarem ações de saúde.

O machismo não só afeta as mulheres como também aos homens, Feitosa et al. 2019 adiciona a problemática de que o sexo masculino não adere tão facilmente a prevenção e promoção de saúde e que isso advém de um conceito antigo de que o homem não precisa cuidar da saúde, para destacar assim sua masculinidade. Ao contrário da mulher o homem não tem sua sexualidade reprimida ao longo de sua vida, inclusive é estimulada pela sociedade e no próprio ambiente familiar, diante disso o homem tende a não enxergar a prevenção e promoção de saúde como algo necessário já que se baseia na premissa que para ser considerado um homem viril não precisa de assistência.

Já quando tratamos sobre vacinação na adolescência, é preciso haver muito cuidado na forma de abordar o assunto, pois ainda é uma temática polêmica onde muitas famílias ainda relutam em aceitar por terem pensamentos errôneos sobre a real finalidade da vacina. Os responsáveis na maioria das vezes não possuem instrução adequada de como abordar esse assunto com os adolescentes e diante disso é possível observar a necessidade de instrução para os pais sendo eles na maioria das vezes a principal rede de apoio dos jovens. $\mathrm{O}$ assunto de sexualidade não deve ser tratado como tabu e deve ser cuidadosamente abordado com crianças e adolescentes, de forma diferenciada para cada idade, pois mediante uma educação sexual adequadas pode-se evitar problemas como o abuso sexual, sexarca precoce e ISTs. 
Os serviços de saúde são locais privilegiados para a promoção da saúde onde os profissionais são tidos como referências. No entanto, se no atendimento à mulher ficar implícito alguma crítica ou julgamento por parte do profissional de saúde, pode-se perder a chance de criar um vínculo com essa mulher e possivelmente educá-la, o que pode levar a um possível abandono de tratamento dessa mulher na UBS. Toda a equipe dos serviços de saúde, em especial o profissional enfermeiro, deve estar sensibilizada quanto aos direitos sexuais das mulheres levando em conta particularidades como religião, família, e a realidade biopsicossocial onde essa mulher se encontra.

\subsection{Atuação da Enfermagem frente às condições emocionais relacionadas ao HPV}

Dalmacio et al. 2019 disserta sobre os aspectos emocionais relacionados ao diagnóstico de HPV serem discutidos e colocados em pauta pelos profissionais da saúde. Muitas mulheres sentem sentimentos negativos como vergonha, tristeza, negação, preocupação, medo e culpa referente a infecção e o que ela pode acarretar para seu futuro, principalmente quando há uma associação do HPV ao CCU. O autor também enfatiza em seu estudo o medo do desconhecido, enfatizando a importância de tornar o assunto amplamente discutido, para que mais mulheres possam se apoiar compartilhando experiências, a fim de minimizar preconceitos e maximizar saberes. $\mathrm{O}$ autor ainda acrescenta um questionamento sobre o que as mulheres entendem como saúde, visto que se dizem em boas condições de saúde porém apresentam uma variedade de sentimentos negativos.

De acordo com a OMS, o conceito de saúde não permeia mais aquele arcaico modelo biomédico e sim todos os aspectos que se relacionam com as necessidades humanas básicas, as quais norteiam uma importante teoria de enfermagem: O princípio das necessidades humanas básicas de Wanda Horta (1979). Essa teoria estabelece a classificação de necessidades, sendo elas: psicobiológicas (fisiologia e segurança), psicossociais (estima, amor/relacionamento) e psicoespirituais (realização pessoal). Ao analisar essa teoria, foi possível entender que a infecção pelo papilomavírus humano interfere em todas as classificações, desde sua fisiologia até a maneira de como essa mulher se enxerga diante da sociedade.

Santo et al, 2013 afirma que sentimentos de medo, vergonha, ou receio podem afetar a confiança e a educação em saúde das mulheres. Sendo listados pelas próprias mulheres, alguns fatores que mais causam desconforto nas consultas, sendo eles: profissional ser do sexo oposto, idade igual ou inferior a da cliente e tempo de experiencia insuficiente na atuação profissional na UBS. Havendo relatos de que indicações de profissionais que realizaram um bom atendimento é um fator que auxilia no conforto do paciente.

Diante dos aspectos emocionais, faz-se necessário uma atuação do enfermeiro para o acolhimento dessas mulheres durante as consultas de enfermagem com o objetivo de estabelecer um local seguro e confortável para que elas possam expor seus sentimentos, suas preocupações e seu modo de vida (Silva et al. 2013). Conforme afirma a teoria ambientalista de Florence Nightingale (1859), que foca no ambiente da assistência à saúde dos indivíduos, a assistência de enfermagem deve ser preferencialmente realizada em um ambiente sanitário adequado, limpo, confiável e organizado. Possibilitando ao enfermeiro atuar com respeito, construindo o vínculo e confiança necessárias para realizar um trabalho empático e humanizado, utilizando a ciência como sua base.

De acordo com a teoria de Hildegard Peplau (1952), o estabelecimento da relação interpessoal da enfermagem com a paciente é importante para que as necessidades sejam identificadas, as dúvidas sejam sanadas e os vínculos sejam criados. Portanto, a consulta de enfermagem exige do profissional uma postura que preserve a intimidade da cliente possibilitando uma experiência confortável e sem traumas, com o máximo de cautela, sem deixar transparecer opiniões ou críticas, criando assim um ambiente amigável e seguro.

Carvalho et al. 2017, também propõe incorporar um cuidado humanizado, compartilhando informações de modo que atenda as demandas daquela mulher adaptando a fala de acordo com a realidade de cada uma. É válido utilizar estratégias focadas 
em aproximar a rede de apoio para que a mulher receba o apoio emocional não só na unidade de saúde, mas também em seu lar. (Dalmacio et al. 2019)

Em comparação, Santos, et al. 2013, relata em seu estudo que essa estratégia é pouco implementada por profissionais na UBS, proporcionando um tratamento incompleto e não satisfatório, o qual pode ocasionar em abandono do tratamento das mulheres na UBS, atingindo assim a reputação do Sistema Único de Saúde (SUS). Para que haja um efetivo tratamento e uma efetiva abordagem para com as pacientes, Silva et al. 2013 propõe um atendimento de forma integral e não baseada na abordagem sindrômica, pois esta pode contribuir para a fragmentação do cuidado e não engloba uma visão holística. Por isso, na atenção básica precisa haver sempre a interdisciplinaridade de profissionais, buscando constantemente estimular o autocuidado do paciente.

A Teoria do autocuidado de Dorothea Orem (1971) é bastante utilizada para conduzir estratégias de orientação e promoção do autocuidado com sentido de garantir que o tratamento seja eficaz e para que assim haja uma melhor qualidade de vida. Por meio desta Teoria, foram identificados no estudo de Dalmacio et al. 2019, alguns fatores que limitam o autocuidado, que são, o déficit de conhecimento em relação à doença, poucas ações de autocuidado realizadas e seus sentimentos em relação ao HPV. O autor articula que para favorecer o autocuidado e fortalecer a relação interpessoal, a orientação através de ações da enfermagem auxiliam a mulher a potencializar seus níveis de autocuidado. Esta teoria é salientada por Silva, et al. 2013, que também considera que o envolvimento das mulheres no processo de educação em saúde pode ser maximizado por tais ações de enfermagem.

\section{Conclusão}

Ao examinar os artigos selecionados, evidenciamos que as ações desenvolvidas pelo profissional enfermeiro são de extrema importância para prevenção do HPV na atenção básica e contribuem para assegurar uma melhor prática da Sistematização da Assistência da Enfermagem, além de estimular a educação em saúde, realizando a promoção e prevenção.

Ficou evidente que o cuidado das mulheres com o HPV na atenção básica é a consequência de um atendimento com acolhimento ineficaz e uma baixa propagação de informações, expressando falhas na gestão, problemas para estabelecer um fluxo assistencial adequado, necessidade de novas técnicas preventivas e educativas e educação continuada para os profissionais de saúde. A falta de continuidade do tratamento das mulheres pode estar diretamente relacionada à falta de acolhimento e escuta qualificada como também à falta de profissionais nas UBS, o que evidencia os empecilhos para uma gestão de qualidade.

Dessa maneira, destaca-se a importância de uma consulta de enfermagem com respeito e empatia além da criação de novas estratégias de propagação de informações, como a utilização das redes sociais e a inclusão de ações de educação em saúde em escolas e igrejas, para que assim possa-se alcançar o público feminino com maior efetividade. Verifica-se que a escassez de políticas públicas que incentivem a prevenção do HPV e estimulem a vacinação dos adolescentes como também uma carência de educação em saúde sexual por parte da população feminina, as quais são as mais afetadas pelo HPV, como fatores limitante da atuação do enfermeiro na atenção básica.

Sendo assim, é necessário atentar-se aos fatores de risco, prevenção e promoção da saúde relacionados ao HPV, bem como à orientação sexual e aos tabus sociais associados à sexualidade feminina. Fica evidente que o enfermeiro tem importante papel na prevenção do HPV na atenção básica ao ter protagonismo na formação de vínculo, ao realizar a escuta ativa e busca ativa, praticando empatia e humanização em todos os aspectos das consultas para que as mesmas aconteçam de forma holística, com relacionamento interpessoal adequado e ambiente seguro. 
Espera-se que outros estudos sejam conduzidos com o propósito de levantar discussões ainda pouco ponderadas na prevenção do HPV e que busquem detectar novas estratégias para aprimorar a atuação da enfermagem a fim de respaldar a sua importância acerca da temática.

\section{Referências}

Associação Hospitalar Moinhos de Vento (2017). Estudo Epidemiológico sobre a Prevalência Nacional de Infecção pelo HPV (POP-Brasil): Resultados preliminares. http://www.iepmoinhos.com.br/pesquisa/downloads/LIVRO-POP_Brasil_-_Resultados_Preliminares.pdf

Brasil. (2012). Ministério da Saúde. Secretaria de Atenção à Saúde. Departamento de Atenção Básica. Política Nacional de Atenção Básica. http://bvsms.saude.gov.br/bvs/publicacoes/politica_nacional_atencao_basica.pdf

Brasil. (2013). Ministério da saúde. Secretaria de Atenção à Saúde Departamento de Atenção Básica. Cadernos de Atenção Básica, Controle dos cânceres de colo do útero e da mama. https://bvsms.saude.gov.br/bvs/publicacoes/controle_canceres_colo_utero_2013.pdf

Brasil. (2014). Ministério da saúde. Secretaria de vigilância em saúde departamento de vigilância de doenças transmissíveis coordenação-geral do programa nacional de imunizações. Informe técnico sobre a vacina papilomavírus humano (hpv) na atenção básica. https://portalarquivos2.saude.gov.br/images/pdf/2015/junho/26/informe-t--cnico-introdu----o-vacina-hpv-18-2-2014.pdf

Brasil. (2015). Ministério da Saúde. Secretaria de Vigilância em Saúde. Departamento de Doenças de Condições Crônicas e Infecções Sexualmente Transmissíveis. Protocolo Clínico e Diretrizes Terapêuticas para Atenção Integral às Pessoas com Infecções Sexualmente Transmissíveis (IST). https://bvsms.saude.gov.br/bvs/publicacoes/protocolo_clinico_diretrizes_terapeutica_atencao_integral_pessoas_infeccoes_sexualmente_transmissiveis.pdf

Brasil. (2016). Ministério da saúde. Instituto Nacional de Câncer (INCA). Diretrizes Brasileiras para o rastreamento Do Câncer do Colo do Útero. https://www.inca.gov.br/sites/ufu.sti.inca.local/files//media/document//diretrizesparaorastreamentodocancerdocolodoutero_2016_corrigido.pdf

Carvalho, M., Queiroz, A., Moura, M., Marques, S., Vieira, B., \& Ferreira, D. (2017). Fatores de risco de mulheres adolescentes e jovens frente ao Papilomavírus Humano [Human Papilloma Virus-related risk factors for adolescent and young women] [Factores de riesgo para las adolescentes y jóvenes mujeres ante el Virus del Papiloma Humano]. Revista Enfermagem UERJ, 25, e25823. https://doi.org/10.12957/reuerj.2017.25823

Cestari, Maria Elisa Wotzasek et al. Necessidades de cuidados de mulheres infectadas pelo papilomavírus humano: uma abordagem compreensiva. Revista da Escola de Enfermagem da USP, 46(5), 1082-1087. https://doi.org/10.1590/S0080-62342012000500007

Conceição, J. P. S., Medeiros, M. M. da S., Rodrigues, L. M. S., Bráz, M. R., Balbino, C. M., \& Silvino, Z. R. (2017). O conhecimento do enfermeiro sobre a prevenção do câncer de colo de útero na atenção básica*. Revista Enfermagem Atual In Derme, 2017. https://doi.org/10.31011/reaid-2017-v.2017-n.0-art.552

Da Silva, M., Gitsos, J., \& dos Santos, N. (2014). Atenção básica em saúde: prevenção do câncer de colo do útero na consulta de enfermagem [Primary health care: cervical cancer prevention in nursing Consultation]. Revista Enfermagem UERJ, 21(5), 631-636. https://www.epublicacoes.uerj.br/index.php/enfermagemuerj/article/view/10039

Dalmacio, N., Costa, B., Souza, S., \& Aguiar, V. (2019). Percepção da mulher com hpv e seu autocuidado. Revista de Enfermagem UFPE on line, 13. https://doi.org/10.5205/1981-8963.2019.237305

Dantas, P., Leite, K., César, E., Silva, S., Souza, T., \& Nascimento, B. (2018). Conhecimento das mulheres e fatores da não adesão acerca do exame papanicolau. Revista de Enfermagem UFPE on line, 12(3), 684-691. https://doi.org/10.5205/1981-8963-v12i3a22582p684-691-2018

De Oliveira, V. C, da Silva, M. R, Viegas, S. M. F, Guimarães, E. A. A, da Fonseca, D. F., \& de Oliveira, P. P. (2019). Vivência de responsáveis por adolescentes na vacinação contra o papilomavírus: estudo fenomenológico. Online braz. j. nurs. (Online), 18(2). https://doi.org/10.17665/1676-4285.20195985

De Souza, A. F., \& Costa, L. H. R. (2015). Conhecimento de Mulheres sobre HPV e Câncer do Colo do Útero após Consulta de Enfermagem. Revista Brasileira De Cancerologia, 61(4), 343-350. https://doi.org/10.32635/2176-9745.RBC.2015v61n4.220

Feitosa, L., Alves, D., Pereira, E., Nunes, V., Hipólito, U., \& Almeida, M. (2019). Imunização contra papilomavirus humano em escolas municipais. Revista de Enfermagem UFPE on line, 13. https://doi.org/10.5205/1981-8963.2019.241812

Guedes, M., São Bento, P., Telles, A., Queiroz, A., \& Xavier, R. (2016). A vacina do papilomavírus humano e o câncer do colo do útero: uma reflexão. Revista de Enfermagem UFPE on line, 11(1), 224-231. https://doi.org/10.5205/1981-8963-v11i1a11897p224-231-2017

H C. K., Sholtis B. L., \& Smith S. D. (2014). Brunner \& Suddarth. Tratado de Enfermagem Médico-Cirúrgica, (12a ed.).

Horta, W. A. (1979). Processo de enfermagem. E.P.U

Instituto Nacional de Câncer José Alencar Gomes da Silva (2019). Estimativa 2020: incidência de câncer no Brasil. https://www.inca.gov.br/sites/ufu.sti.inca.local/files/media/document/estimativa-2020-incidencia-de-cancer-no-brasil.pdf

Mendes, K. D. S., Silveira, R. C. C. P., \& Galvão, C. M. (2008). Revisão integrativa: método de pesquisa para a incorporação de evidências na saúde e na enfermagem. Texto Contexto Enferm, 17(4): 758-64. https://doi.org/10.1590/S0104-07072008000400018

Nogueira da Silva, P. L. N., Martins, F. G. S., Galvão, A. P. F. C., Souto, S. G. T., Oliveira, R. S., \& Martins, I. M. L. (2021). Sentimentos de pré-adolescentes e adolescentes quanto à vacinação contra o papilomavirus humano. Revista Nursing 24(273): 5299-5310. https://doi.org/10.36489/nursing.2021v24i273p5299. 5310 
Research, Society and Development, v. 10, n. 11, e106101119271, 2021

(CC BY 4.0) | ISSN 2525-3409 | DOI: http://dx.doi.org/10.33448/rsd-v10i11.19271

Rodrigues da Silva, L., Cordeiro, E., da Silva, T., Daltro Da Silva Albuquerque, A., Rodrigues Ferreira, M., \& Leite da Silva, T. (2016). Adesão ao exame Papanicolau por mulheres jovens em unidade básica de saúde. Revista de Enfermagem UFPE on line, 10(12), 4637-4645.https://doi.org/10.5205/1981-8963v10i12a11533p4637-4645-2016

Santiago, T., Andrade, M., \& do Nascimento Paixão, G. (2015). Conhecimento e prática das mulheres atendidas na unidade de saúde da família sobre o Papanicolaou [Knowledge and practice on Pap smear tests by women assisted at family health units]. Revista Enfermagem UERJ, 22(6), 822-829. https://doi.org/10.12957/reuerj.2014.6368

Santos, L., Dorcas de Melo Inagaki, A., Freire Abud, A., Katrin Albuquerque de Oliveira, J., Nunes Ribeiro, C., \& Alves de Oliveira, M. (2014). Características sociodemográficas e risco para doenças sexualmente transmissíveis entre mulheres atendidas na atenção básica [Sociodemographic characteristics and risk factors for sexually transmitted diseases among women assisted at primary care unit]. Revista Enfermagem UERJ, 22(1), 111-115. https://www.epublicacoes.uerj.br/index.php/enfermagemuerj/article/view/11456/8992

Santos, U. M., \& de Souza, S. E. B. (2013). Papanicolau: diagnóstico precoce ou prevenção do câncer cervical uterino? Revista Baiana de Saúde Pública, 37(4), 941-951. https://doi.org/10.22278/2318-2660.2013.v37.n4.a420 